\title{
A candidate gene investigation of methylphenidate response in adult attention-deficit/hyperactivity disorder patients: results from a naturalistic study
}

\author{
Tor-Arne Hegvik $^{1,2}$ - Kaya Kvarme Jacobsen ${ }^{1,2,3} \cdot$ Mats Fredriksen $^{4,5}$ • \\ Tetyana Zayats ${ }^{1,2} \cdot$ Jan Haavik ${ }^{1,2,6}$
}

Received: 21 September 2015/Accepted: 20 March 2016/Published online: 18 April 2016

(c) The Author(s) 2016. This article is published with open access at Springerlink.com

\begin{abstract}
Attention-deficit/hyperactivity disorder (ADHD) is a common childhood onset neuropsychiatric disorder with a complex and heterogeneous symptomatology. Persistence of ADHD symptoms into adulthood is common. Methylphenidate (MPH) is a widely prescribed stimulant compound that may be effective against ADHD symptoms in children and adults. However, MPH does not exert satisfactory effect in all patients. Several genetic variants have been proposed to predict either treatment response or adverse effects of stimulants. We conducted a literature search to identify previously reported variants associated with MPH response and additional variants that were biologically plausible candidates for MPH response. The response to MPH was assessed by the treating clinicians in 564 adult ADHD patients and 20 genetic variants were successfully genotyped. Logistic regression was used to test for association between these polymorphisms and
\end{abstract}

Electronic supplementary material The online version of this article (doi:10.1007/s00702-016-1540-7) contains supplementary material, which is available to authorized users.

Tor-Arne Hegvik

tahegvik@gmail.com

1 Department of Biomedicine, University of Bergen, 5009 Bergen, Norway

2 K.G. Jebsen Centre for Research on Neuropsychiatric Disorders, University of Bergen, 5009 Bergen, Norway

3 Center for Medical Genetics and Molecular Medicine, Haukeland University Hospital, 5021 Bergen, Norway

4 Division of Mental Health and Addiction, Vestfold Hospital Trust, 3101 Tønsberg, Norway

5 University of Oslo, 0318 Oslo, Norway

6 Division of Psychiatry, Haukeland University Hospital, 5021 Bergen, Norway treatment response. Nominal associations $(p<0.05)$ were meta-analysed with published data from previous comparable studies. In our analyses, rs 1800544 in the ADRA2A gene was associated with MPH response at a nominal significance level (OR 0.560, 95\% CI 0.329-0.953, $p=0.033$ ). However, this finding was not affirmed in the meta-analysis. No genetic variants revealed significant associations after correction for multiple testing $(p<0.00125)$. Our results suggest that none of the studied variants are strong predictors of MPH response in adult ADHD as judged by clinician ratings, potentially except for rs1800544. Consequently, pharmacogenetic testing in routine clinical care is not supported by our analyses. Further studies on the pharmacogenetics of adult ADHD are warranted.

Keywords ADHD - Pharmacogenetics - ADRA2A . Methylphenidate $\cdot$ Stimulants

\section{Introduction}

Attention-deficit/hyperactivity disorder (ADHD) is a neuropsychiatric disorder characterized by developmentally inappropriate levels of impulsivity, hyperactivity and inattention (American Psychiatric Association 2013). Most ADHD patients are diagnosed in childhood and the symptoms are thought to persist into adulthood in its full syndromic form in approximately $35 \%$ of the patients. However, more than $70 \%$ of the patients may be burdened by ADHD symptoms or experience ADHD-related functional impairment as adults (Biederman et al. 2010, 2012).

The first-line pharmacological option in the treatment of ADHD in children and adults is methylphenidate (MPH) (National Institute for Health and Clinical Excellence 
2008; Norwegian Directorate of Health 2014). MPH has been shown superior to placebo in providing symptom relief in ADHD (Epstein et al. 2014; Faraone and Buitelaar 2010), and is believed to exert its effect by inhibiting the dopamine transporter (encoded by DAT/SLC6A3 gene) and/ or the noradrenaline transporter (encoded by NET/SLC6A2 gene) (Engert and Pruessner 2008). Although the exact mechanisms of MPH's effects remain unknown, the inhibition of these transporters is thought to affect the extracellular concentrations of dopamine and noradrenaline in the brain, predominantly in the prefrontal cortex and striatum, and thus, moderate the symptoms of ADHD (Arnsten and Pliszka 2011; Engert and Pruessner 2008).

The response rates to MPH among adult ADHD patients range from 25 to $78 \%$ in controlled trials (Wilens et al. 2011). A portion of this variability is likely explained by the definition of response, diagnostic criteria, sample characteristics, drop-outs and dosing parameters (Fredriksen et al. 2013; Wilens et al. 2011). In addition, some patients experience adverse effects that outweigh the therapeutic impact, although serious adverse effects are considered rare (Fredriksen et al. 2013). Genetic variations may contribute to variability in MPH response. Previous studies have investigated several single nucleotide polymorphisms (SNPs) and variable tandem repeats (VNTRs), mostly in or near genes involved in the dopamine and noradrenaline systems, as well as in genes encoding MPH metabolizing enzymes (Bruxel et al. 2014). Still, most reports have been negative for any association, or the results have been conflicting (Bruxel et al. 2014; Kambeitz et al. 2014).

One explanation for the lack of consistent pharmacogenetic findings could be the polygenic and multifactorial nature of ADHD, with genetic variants contributing small effects to its aetiology and, consequently, requiring large sample sizes in order to detect such effects. An additional potentially important factor may be the phenotypic heterogeneity of ADHD. Moreover, the symptoms of ADHD tend to change with age with most studies reporting a more pronounced decline in the hyperactivity and impulsivity symptoms, as compared to the inattention symptoms (Biederman et al. 2010, 2012; Pingault et al. 2015). MPH may not be effective against all ADHD symptoms to the same extent and the effect of MPH may change over time. Thus, pharmacogenetic studies conducted in ADHD children may not be directly applicable to adults. Despite this lack of reliable genetic markers in the published literature and the uncertainties regarding the impact of aging, several commercial genetic tests are being marketed to patients, parents and health care providers to guide pharmacological intervention in ADHD. Examples of such commercial tests include "Harmonyx ${ }^{\circledR}$ Test for
ADHD" (Harmonyx Diagnostics) and "GeneSight ${ }^{\circledR}$ ADHD” (Assurex Health).

Discovering and affirming genetic variants involved in the response to MPH in ADHD patients could help guide treatment strategies with respect to symptom control and adverse effects, thus helping to secure treatment compliance and adherence (Gajria et al. 2014). This may be of great importance for adult ADHD patients, who often are burdened with polypharmacy, comorbidity and socioeconomic distress (Halmoy et al. 2009). In addition, identification of pharmacogenetic variants could provide greater insight into the pharmacodynamics of MPH and the biology of ADHD. In this study, we therefore, explored MPH response in an adult ADHD sample in the light of genetic variants that have previously been associated with changes in MPH response. We also hypothesized that variants in the genes formerly linked to psychiatric or pharmacological phenotypes and which are involved in MPH metabolism or MPH pharmacodynamics, could potentially affect the response to MPH in adult ADHD patients.

\section{Patients and methods}

\section{Patient recruitment and assessment of MPH response}

Participants were recruited as part of a large study on adult ADHD risk factors (Halmoy et al. 2009; Johansson et al. 2008). DNA was extracted from blood or saliva samples collected from clinically diagnosed adult ADHD patients of Norwegian descent all over Norway. The diagnosis of ADHD in adults was based on the Diagnostic and Statistical Manual of Mental Disorders IV (DSM-IV) criteria. The recruitment protocol has previously been described in detail elsewhere (Halmoy et al. 2009; Johansson et al. 2008).

The patients' response to MPH was assessed by two questionnaires that were filled out by the patients' treating physicians, mainly psychiatrists (but also some general practitioners). The questions were designed to reflect treatment decisions in routine clinical settings.

In Questionnaire 1, the physicians rated the effect of "Ritalin" and/or "Concerta" which represented immediate release MPH and extended release MPH, respectively (at the time Questionnaire 1 was made and first utilized, "Ritalin" was the only immediate release formulation and "Concerta" the only extended release formulation of MPH available in Norway). The treatment response was rated with the options: "Very good", "Good", "Has had effect, but discontinued due to side effects" and "Not sure or none". The first three categories were grouped as MPH 
responders and "Not sure or none" were defined as MPH non-responders. Patients who had tried both "Ritalin" and "Concerta", had to be rated as "Not sure or none" on both items to be defined as MPH non-responders. A minority of the patients $(13 \%)$ were scored on a slightly modified version of the questionnaire, Questionnaire 2, where the effect of MPH was rated directly and the response option "Has had effect, but discontinued due to side effects" was omitted. Here, the first two alternatives were used to define MPH responders and the third non-responders.

The physicians filling out the questionnaires also stated the patients' date of birth and the date the questionnaires were signed. These data were used to calculate the age of the patients at the time of questionnaire submission.

The original questionnaire (Questionnaire 1) was developed, and later revised (Questionnaire 2), by the last author of this study, Jan Haavik, together with co-workers during the planning of the larger study on ADHD risk factors (Halmoy et al. 2009; Johansson et al. 2008).

\section{Selection of genetic variants}

To identify genetic polymorphisms (SNPs and VNTRs) previously associated, either experimentally or theoretically, with MPH response in child or adult ADHD patients, we conducted a literature search in PubMed, The Pharmacogenomics Knowledge Base and Google Scholar. In addition, variants in genes involved in ADHD pathophysiology or MPH metabolism and shown to have pharmacogenetic effects were included.

\section{Genotyping and its quality control}

All SNPs were genotyped at CIGENE facility at the Norwegian University of Life Sciences ( $⿱$ As, Norway), with a Sequenom MassARRAY iPLEX system (Sequenom, San Diego, CA, USA) as described previously (Johansson et al. 2010). Variants failing genotyping with the Sequenom MassARRAY iPLEX system, were genotyped as part of a larger genotyping effort using Illumina HumanExome BeadChip 12v1_B (Zayats et al. manuscript in preparation). VNTRs were genotyped by polymerase chain reaction followed by fragment analysis as previously described (Johansson et al. 2008).

The VNTR alleles were dichotomized based on the literature: a specific number of repeats was defined as the risk allele and all others as non-risk. For DRD5, the148 bprepeat allele was defined as the risk allele (Tahir et al. 2000), the 10-repeat allele (10R) was defined as the risk allele for SLC6A3/DAT1 (Kambeitz et al. 2014) and the 7-repeat allele (7R) as the risk allele for DRD4 (Hamarman et al. 2004).
In the analyses presented, genotyped SNPs passed quality control criteria of minor allele frequency (MAF) over $1 \%$, missingness below $10 \%$ and Hardy-Weinberg equilibrium test $p$ value above 0.005 . SNP genotype missingness per individual was set to $15 \%$.

All included VNTRs demonstrated MAF over $1 \%$, missingness below $1 \%$ and passed Hardy-Weinberg equilibrium test $p$ value above 0.005 after dichotomization. VNTR genotype missingness per individual was set to a maximum of one out of three variants.

\section{Genetic association between selected genetic variants and MPH response}

Genetic association between chosen genetic variants and MPH response was tested in the form of binary logistic regression implemented in R-software. Sex and age were included as covariates. Both dominant and additive models were examined. Multiple testing was controlled by Bonferroni method.

\section{Meta-analysis with previous studies}

Genetic variants exhibiting signs of association $(p<0.05)$ were further explored in meta-analysis with the previously published studies. For this step, published data was screened with regard to comparability, meaning studies on adult ADHD patients (17 years of age or more) of European ethnicity with MPH response assessed with a binary outcome and the variants of interest having a similar allele distribution to our material.

Once publications suitable for meta-analysis were identified, reported genetic data were re-examined for association with MPH response together with our data by means of Fisher's exact test. Meta-analysis was carried out using inverse-variance method in a random effects model directly from the counts data. Between-study heterogeneity was assessed by $I^{2}$ measure. Analyses were performed in R-software, using Metafor 1.9-4 package (Viechtbauer 2010). Multiple testing was controlled by Bonferroni method.

\section{Results}

\section{Selection of genetic variants, genotyping and its quality control}

Overall, 20 genetic variants were selected: 17 SNPs and 3 VNTRs in 11 genes (Supplementary Table 1 describes the polymorphisms). All were successfully genotyped. 
Table 1 Description of the examined sample of adult ADHD patients

\begin{tabular}{llll}
\hline & Questionnaire 1 & Questionnaire 2 & Total \\
\hline $\begin{array}{l}\text { Demographics } \\
\text { Sex: male/female }\end{array}$ & $247 / 246$ & & \\
Mean age (SD) & $34.0(10.1)$ & $24 / 47$ & $271 / 293$ \\
MPH effect & & $34.9(9.7)$ & $34.11(10.0)$ \\
Responder (\%) & $427(86.6)$ & $60(84.5)$ & $487(86.3)$ \\
Non-responder $(\%)$ & $66(13.4)$ & $11(15.5)$ & $77(13.7)$ \\
Total & 493 & 71 & 564 \\
\hline
\end{tabular}

$M P H$ methylphenidate, $S D$ standard deviation

Questionnaire 1: Patients with MPH response rated as "Very good", "Good" or "Has had effect, but discontinued due to side effects" defined Responders. "Not sure or none" defined Non-responder

Questionnaire 2: Patients with MPH response rated as "Very good" or "Good" defined Responders. "Not sure or none" defined Non-responders
After quality control, there were 564 unique patients, of whom 487 were classified as MPH responders and 77 as MPH non-responders (Table 1). The number of patients for the different genotyping platforms is presented in Supplementary Table 2.

\section{Genetic association between selected genetic variants and MPH response}

All results of the association analyses are presented in Supplementary Table 3. In summary, prior to correction for multiple testing, one nominally significant $(p<0.05)$ finding in the alpha-2-adrenergic receptor $2 \mathrm{~A}$ gene (ADRA2A) was noted. Namely, the $\mathrm{G}$ allele of rs1800544 (MspI $-1291 \mathrm{C}>\mathrm{G}$ ), located in the 5-untranslated region $\left(5^{\prime} \mathrm{UTR}\right)$, was associated with MPH non-response in a dominant model (OR 0.560, $95 \%$ CI $0.329-0.953$, $p=0.033$ ).

No genetic variations were associated with MPH response after correction for multiple testing $(p<0.00125)$.

\section{Meta-analysis with previous studies}

In total, one variant in a dominant genetic model, rs1800544, was associated with MPH response prior to Bonferroni correction for multiple testing and included in the meta-analysis. After screening the previously identified literature with our criteria, only one study was performed on adult ADHD patients of European descent with dichotomous assessment of MPH response (Contini et al. 2011) and, thus, suitable for meta-analysis together with our data. The threshold of significance was set to $p<0.00122$.

The meta-analysis showed no significant effect of rs 1800544 on MPH response (OR 0.711, $95 \%$ CI $0.410-1.232, p=0.251)$ (Table 2).

\section{Discussion}

Knowledge about the pharmacogenetics of MPH response may increase our insights into the aetiology of ADHD and improve patient care. We therefore assessed the genetic background of MPH response in patients with adult ADHD.

One SNP, rs 1800544 , in the $5^{\prime} \mathrm{UTR}$ of the $A D R A 2 A$ gene revealed nominal association with MPH response in adult ADHD patients. Located in a transcription factor binding site, rs1800544 may affect gene expression and, consequently, MPH's pharmacological effect on the symptoms of ADHD (Xu and Taylor 2009). ADRA2A encodes an adrenergic receptor located pre- and post-synaptically in both the central and peripheral nervous system. It has been found to be highly expressed in brain areas that have been associated with ADHD (Arnsten and Pliszka 2011). In addition, electrophysiological studies have shown that MPH may affect cortical excitability through ADRA2A, which might partly explain MPHs effect on the symptoms of ADHD (Andrews and Lavin 2006). The selective ADRA2A agonist guanfacine is used in the treatment of ADHD, consistent with a potential involvement of $A D R A 2 A$ in the pathophysiology of the disorder (Arnsten and Pliszka 2011). It is noteworthy, however, that metaanalysis of this variant with a comparable Brazilian study (Contini et al. 2011), revealed no association. This observation may be explained by a number of reasons: (a) the sample size of the Brazilian study is only approximately one-third of ours, (b) possible genetic admixture as the reported MAF in the Brazilian study is higher than that reported for the CEU population (Northern and Western Europeans from Utah, UT, USA) and more comparable to that of the MXL population (Mexican Ancestry in Los Angeles, CA, USA) (Abecasis et al. 2012) and (c), the phenotypic heterogeneity of ADHD. Thus, meta-analysis with a larger and more homogeneous sample is warranted. 
Table 2 Results of meta-analysis of rs1800544 genotypes and MPH response

\begin{tabular}{llllll}
\hline $\begin{array}{l}\text { Study (observed G-allele } \\
\text { frequency }{ }^{\mathrm{a}} \text { ) }\end{array}$ & $\begin{array}{l}\text { rs1800544 } \\
\text { genotype }\end{array}$ & $\begin{array}{l}\text { MPH } \\
\text { responders }\end{array}$ & $\begin{array}{l}\text { MPH non- } \\
\text { responders }\end{array}$ & OR (95 \%CI) & $\begin{array}{l}\text { Crude } \\
p \text { value* }\end{array}$ \\
\hline Contini et al. (0.33) & GG+GC & 67 & 13 & $1.069(0.398-2.824)$ & 1.000 \\
& CC & 53 & 11 & & \\
This study (0.27) & GG+GC & 194 & 39 & $0.587(0.333-1.024)$ & 0.048 \\
& CC & 229 & 27 & $0.711(0.410-1.232)$ & 0.251 \\
Meta-analysis & & & & $24.17 \%$ \\
\hline
\end{tabular}

$\mathrm{OR}>1$ means that the variant is associated with MPH response and OR $<1$ that the variant is associated with MPH non-response

$M P H$ methylphenidate, $O R$ odds ratio, $95 \%$ CI $95 \%$ confidence interval, $I^{2}$ heterogeneity measure

* Threshold for significance after $40+1$ tests $p=0.00122$

${ }^{\text {a }}$ In both MPH responders and MPH non-responders

Previously, the $\mathrm{G}$ allele of rs 1800544 has been noted to be associated with the improvement of MPH response in children and adolescents (Cheon et al. 2009; da Silva et al. 2008; Polanczyk et al. 2007). However, negative studies have also been reported in children and adolescents (Kim et al. 2011; Park et al. 2013) as well as in adults (Contini et al. 2011). Interestingly, in our study of adult ADHD, the $\mathrm{G}$ allele was associated with MPH non-response. As the symptomatology of ADHD is thought to change with age (Biederman et al. 2010, 2012; Faraone et al. 2006; Pingault et al. 2015), it is difficult to draw firm conclusions regarding the exact effect of this allele. There may be agerelated changes in human neurobiology, such as alterations in gene expression patterns (Shingai et al. 2014).

In general, pharmacogenetic findings in paediatric ADHD samples reveal little to no replication in adults. This absence of replication may be explained by age-related changes in ADHD symptomatology, as well as likely variability in MPH effect itself. It has been reported in some studies that, compared to inattention, MPH has greater impact on the symptoms of hyperactivity and impulsivity in children (Beery et al. 2013). As inattention is believed to become more pronounced in the adult form of ADHD, pharmacological effects of MPH on attention may be more important in this group. Consequently, the genetic variants mediating the pharmacogenetic effects of MPH seen in children may not be of the same importance in adults. Nonetheless, randomised controlled studies in adults indicate that MPH is as effective on inattention symptoms as it is on hyperactivity and impulsivity (Epstein et al. 2014; Spencer et al. 2005).

Pharmacogenetic testing is being introduced for a number of neuropsychiatric disorders, including ADHD (Assurex Health; Dediemar 2015; Harmonyx Diagnostics; Hicks et al. 2015). Based on our results, such testing in adult ADHD for any of the variants assessed in our study is not justified, as only a single variant was nominally associated with MPH response. Additionally this effect was in the opposite direction of what has previously been described in studies conducted in children and adolescents.

This study should be viewed in the light of its limitations. Even with 564 participants, which to our knowledge is the largest pharmacogenetic study in ADHD, our study is still small and relatively underpowered to detect the small effect sizes of common variants.

Another limitation of our study may be phenotypic heterogeneity. Since psychiatric comorbidities, including substance abuse as well as polypharmacy, are common among our recruited adult ADHD patients (Halmoy et al. 2009), the MPH response observed in this study is likely to be affected by these factors (Retz and Retz-Junginger 2014). However, the presence of comorbidities may better approximate the average ADHD patient encountered in routine clinical practice (Sobanski et al. 2007). Furthermore, a recent naturalistic survey on instant release MPH concluded that psychiatric comorbidities may not be important predictors of medication response (Victor et al. 2014).

The overall treatment response was assessed by clinicians using two slightly different variants of a questionnaire. It is unlikely that there were systematic differences in how the physicians filled out the questionnaires, as the percentage of treatment responders was very similar in both (Table 1). Moreover, since this is an observational study, observer-expectancy effects might be present. The response rating schemes used in this study were designed to mirror routine clinical judgement, which is usually based on symptom scores and additional information provided by the patients. Thus, such clinical ratings are not directly comparable to the response scores derived from single symptom measurements or neuropsychological tests. In addition, laboratory recorded phenotypes and symptom rating scales may only capture minor phenotype changes and core symptom reduction (Beery et al. 2013). In contrast, our phenotype may better reflect changes in the patients' everyday function. 
A substantial proportion of our sample was recruited through a registry of adult ADHD patients who applied for stimulant treatment (Johansson et al. 2008). Therefore, some recruitment bias could be present in our sample. It may be that our patients had more severe ADHD symptoms as compared to the "average" ADHD patients, and severity has been shown to be a positive predictor of MPH response (Buitelaar et al. 2011; Victor et al. 2014). However, the patients with the greatest symptom burden are also the most functionally impaired, and would therefore perhaps be the ones that could benefit the most from accurate genetic guidance of pharmacological therapy.

Finally, the strength of the association signals presented in the previous pharmacogenetic studies also affect the outcomes of this study. For instance, as several of the previously reported variants have only been tested in small and underpowered studies, false positive findings are likely. Moreover, some of the variants investigated in this study were tested for association with clinical response to MPH for the first time.

In summary, none of the examined variants reached the threshold for statistical significance after correction for multiple testing. The SNP rs1800544 in ADRA2A, revealed nominally significant association, but the direction of effect was the opposite to that reported in previous studies conducted in children and adolescents. As some of the examined variants represent the existing pharmacogenetic markers of MPH, also used in commercial tests, our results suggest that such tests cannot reliably predict treatment response in adult ADHD patients. Therefore, more research is warranted before these markers can be recommended for routine clinical use.

\footnotetext{
Acknowledgments First and foremost, we would like to thank our patients for their participation. We are also grateful to the clinicians for their assistance, to Paal Borge for his help with technical tasks, to Lisa Vårdal for her help with administrative tasks and Professor Stefan Johansson for valuable discussions and proof-reading. This study has received financial support from the Kristian Gerhard Jebsen Foundation, Western Norway Regional Health Authorities and the University of Bergen.
}

\section{Compliance with ethical standards}

Conflict of interest During the past 3 years Jan Haavik has received speaker fees from Lilly, Novartis and Janssen-Cilag. The other authors have no conflict of interest.

Ethical approval This study has been approved by the Regional Committee for Medical Research Ethics of Western Norway (IRB 00001872). Informed written consent was obtained from all individual participants included in the study.

Open Access This article is distributed under the terms of the Creative Commons Attribution 4.0 International License (http://creative commons.org/licenses/by/4.0/), which permits unrestricted use, distribution, and reproduction in any medium, provided you give appropriate credit to the original author(s) and the source, provide a link to the Creative Commons license, and indicate if changes were made.

\section{References}

Abecasis GR et al (2012) An integrated map of genetic variation from 1092 human genomes. Nature 491:56-65. doi:10.1038/ nature 11632

American Psychiatric Association (2013) Diagnostic and statistical manual of mental disorders, 5th edn

Andrews GD, Lavin A (2006) Methylphenidate increases cortical excitability via activation of alpha- 2 noradrenergic receptors. Neuropsychopharmacology 31:594-601. doi:10.1038/sj.npp. 1300818

Arnsten AF, Pliszka SR (2011) Catecholamine influences on prefrontal cortical function: relevance to treatment of attention deficit/hyperactivity disorder and related disorders. Pharmacol Biochem Behav 99:211-216. doi:10.1016/j.pbb.2011.01.020

Assurex Health GeneSight ADHD. https://genesight.com/clinicians/ genesight-tests/adhd/. Accessed 22 Jan 2016

Beery SH, Quay HC, Pelham WE Jr (2013) Differential response to methylphenidate in inattentive and combined subtype ADHD. J Atten Disord. doi:10.1177/1087054712469256

Biederman J, Petty CR, Evans M, Small J, Faraone SV (2010) How persistent is ADHD? A controlled 10-year follow-up study of boys with ADHD. Psychiatry Res 177:299-304. doi:10.1016/j. psychres.2009.12.010

Biederman J, Petty CR, O’Connor KB, Hyder LL, Faraone SV (2012) Predictors of persistence in girls with attention deficit hyperactivity disorder: results from an 11-year controlled follow-up study. Acta Psychiatr Scand 125:147-156. doi:10.1111/j.16000447.2011.01797.x

Bruxel EM, Akutagava-Martins GC, Salatino-Oliveira A, Contini V, Kieling C, Hutz MH, Rohde LA (2014) ADHD pharmacogenetics across the life cycle: new findings and perspectives. Am J Med Genet Part B Neuropsychiatr Genet 165B:263-282. doi:10. 1002/ajmg.b.32240

Buitelaar JK et al (2011) Predictors of treatment outcome in adults with ADHD treated with OROS(R) methylphenidate. Prog Neuropsychopharmacol Biol Psychiatry 35:554-560. doi:10. 1016/j.pnpbp.2010.12.016

Cheon KA, Cho DY, Koo MS, Song DH, Namkoong K (2009) Association between homozygosity of a $G$ allele of the alpha-2aadrenergic receptor gene and methylphenidate response in Korean children and adolescents with attention-deficit/hyperactivity disorder. Biol Psychiatry 65:564-570. doi:10.1016/j. biopsych.2008.12.003

Contini V et al (2011) Adrenergic alpha2A receptor gene is not associated with methylphenidate response in adults with ADHD. Eur Arch Psychiatry Clin Neurosci 261:205-211. doi:10.1007/ s00406-010-0172-4

da Silva TL, Pianca TG, Roman T, Hutz MH, Faraone SV, Schmitz M, Rohde LA (2008) Adrenergic alpha2A receptor gene and response to methylphenidate in attention-deficit/hyperactivity disorder-predominantly inattentive type. J Neural Transm (Vienna, Austria: 1996) 115:341-345. doi:10.1007/s00702-0070835-0

Dediemar S (2015) Assurex Health Tests 200,000 Patients with GeneSight ${ }^{\circledR}$; Precision medicine test proven to provide healthcare cost savings, improve outcomes. http://www.prweb.com/ releases/2015/09/prweb12982584.htm. Accessed 26 Jan 2016

Engert V, Pruessner JC (2008) Dopaminergic and noradrenergic contributions to functionality in ADHD: the role of methylphenidate. Curr Neuropharmacol 6:322-328. doi:10.2174/ 157015908787386069

Epstein T, Patsopoulos NA, Weiser M (2014) Immediate-release methylphenidate for attention deficit hyperactivity disorder 
(ADHD) in adults. Cochrane Database Syst Rev 9:CD005041. doi:10.1002/14651858.CD005041.pub2

Faraone SV, Biederman J, Mick E (2006) The age-dependent decline of attention deficit hyperactivity disorder: a meta-analysis of follow-up studies. Psychol Med 36:159-165. doi:10.1017/ s003329170500471x

Faraone SV, Buitelaar J (2010) Comparing the efficacy of stimulants for ADHD in children and adolescents using meta-analysis. Eur Child Adolesc Psychiatry 19:353-364. doi:10.1007/s00787-0090054-3

Fredriksen M, Halmoy A, Faraone SV, Haavik J (2013) Long-term efficacy and safety of treatment with stimulants and atomoxetine in adult ADHD: a review of controlled and naturalistic studies. Eur Neuropsychopharmacol 23:508-527. doi:10.1016/j.euro neuro.2012.07.016

Gajria K, Lu M, Sikirica V, Greven P, Zhong Y, Qin P, Xie J (2014) Adherence, persistence, and medication discontinuation in patients with attention-deficit/hyperactivity disorder-a systematic literature review. Neuropsychiatr Dis Treat 10:1543-1569. doi:10.2147/ndt.s65721

Halmoy A, Fasmer OB, Gillberg C, Haavik J (2009) Occuptional outcome in adult ADHD: impact of symptom profile, comorbid psychiatric problems, and treatment. A cross-sectional study of 510 clinically diagnosed adult ADHD patients. J Atten Disord 13:157-187

Hamarman S, Fossella J, Ulger C, Brimacombe M, Dermody J (2004) Dopamine receptor 4 (DRD4) 7-repeat allele predicts methylphenidate dose response in children with attention deficit hyperactivity disorder: a pharmacogenetic study. J Child Adolesc Psychopharmacol 14:564-574. doi:10.1089/cap.2004.14. 564

Harmonyx Diagnostics Harmonyx ADHD Medication Patient Testing. http://www.harmonyxdiagnostics.com/physician/which-pre scriptions-are-covered-by-the-harmonyx-test/adhd/. Accessed 22 Jan 2016

Hicks JK, Clinical Pharmacogenetics Implementation Consortium (CPIC) et al (2015) Guideline for CYP2D6 and CYP2C19 genotypes and dosing of selective serotonin reuptake inhibitors. Clin Pharmacol Ther 98:127-134. doi:10.1002/cpt.147

Johansson S et al (2008) Genetic analyses of dopamine related genes in adult ADHD patients suggest an association with the DRD5microsatellite repeat, but not with DRD4 or SLC6A3 VNTRs. Am J Med Genet Part B Neuropsychiatr Genet 147B:1470-1475. doi:10.1002/ajmg.b.30662

Johansson S et al (2010) Common variants in the TPH1 and TPH2 regions are not associated with persistent ADHD in a combined sample of 1636 adult cases and 1923 controls from four European populations. Am J Med Genet Part B Neuropsychiatr Genet 153B:1008-1015. doi:10.1002/ajmg.b.31067

Kambeitz J, Romanos M, Ettinger U (2014) Meta-analysis of the association between dopamine transporter genotype and response to methylphenidate treatment in ADHD. Pharmacogenom J 14:77-84. doi:10.1038/tpj.2013.9

Kim BN et al (2011) Val/Val genotype of brain-derived neurotrophic factor (BDNF) Val(6)(6)Met polymorphism is associated with a better response to OROS-MPH in Korean ADHD children. Int $\mathbf{J}$ Neuropsychopharmacol 14:1399-1410. doi:10.1017/ s146114571100099x

National Institute for Health and Clinical Excellence (2008) Attention deficit hyperactivity disorder: diagnosis and management of
ADHD in children, young people and adults. The British Psychological Society and The Royal College of Psychiatrists, Leicester

Norwegian Directorate of Health (2014) ADHD/hyperkinetisk forstyrrelse-nasjonal faglig retningslinje for utredning, behandling og oppfølging. Rett diagnose-individuell behandling. Norwegian Directorate of Health

Park S, Kim JW, Kim BN, Hong SB, Shin MS, Yoo HJ, Cho SC (2013) No significant association between the alpha-2A-adrenergic receptor gene and treatment response in combined or inattentive subtypes of attention-deficit hyperactivity disorder. Pharmacopsychiatry 46:169-174. doi:10.1055/s-0033-1343485

Pingault JB, Viding E, Galera C, Greven CU, Zheng Y, Plomin R, Rijsdijk F (2015) Genetic and environmental influences on the developmental course of attention-deficit/hyperactivity disorder symptoms from childhood to adolescence. JAMA Psychiatry 72:651-658. doi:10.1001/jamapsychiatry.2015.0469

Polanczyk G, Zeni C, Genro JP, Guimaraes AP, Roman T, Hutz MH, Rohde LA (2007) Association of the adrenergic alpha2A receptor gene with methylphenidate improvement of inattentive symptoms in children and adolescents with attention-deficit/ hyperactivity disorder. Arch Gen Psychiatry 64:218-224. doi:10. 1001/archpsyc.64.2.218

Retz W, Retz-Junginger P (2014) Prediction of methylphenidate treatment outcome in adults with attention-deficit/hyperactivity disorder (ADHD). Eur Arch Psychiatry Clin Neurosci 264:S35S43. doi:10.1007/s00406-014-0542-4

Shingai Y, Tateno A, Arakawa R, Sakayori T, Kim W, Suzuki H, Okubo Y (2014) Age-related decline in dopamine transporter in human brain using PET with a new radioligand [(1)(8)F]FEPE2I. Ann Nucl Med 28:220-226. doi:10.1007/s12149-0130798-1

Sobanski E et al (2007) Psychiatric comorbidity and functional impairment in a clinically referred sample of adults with attention-deficit/hyperactivity disorder (ADHD). Eur Arch Psychiatry Clin Neurosci 257:371-377. doi:10.1007/s00406-0070712-8

Spencer T et al (2005) A large, double-blind, randomized clinical trial of methylphenidate in the treatment of adults with attentiondeficit/hyperactivity disorder. Biol Psychiatry 57:456-463. doi:10.1016/j.biopsych.2004.11.043

Tahir E, Yazgan Y, Cirakoglu B, Ozbay F, Waldman I, Asherson PJ (2000) Association and linkage of DRD4 and DRD5 with attention deficit hyperactivity disorder (ADHD) in a sample of Turkish children. Mol Psychiatry 5:396-404

Victor MM et al (2014) Severity but not comorbidities predicts response to methylphenidate in adults with attention-deficit/ hyperactivity disorder: results from a naturalistic study. J Clin Psychopharmacol 34:212-217. doi:10.1097/jcp. 0000000000000091

Viechtbauer W (2010) Conducting meta-analyses in R with the metafor package. J Stat Softw 36:1-48

Wilens TE, Morrison NR, Prince J (2011) An update on the pharmacotherapy of attention-deficit/hyperactivity disorder in adults. Expert Rev Neurother 11:1443-1465. doi:10.1586/ern.11. 137

Xu Z, Taylor JA (2009) SNPinfo: integrating GWAS and candidate gene information into functional SNP selection for genetic association studies. Nucleic Acids Res 37:W600-W605. doi:10. 1093/nar/gkp290 